\title{
O Pesquisador e a Experiência de Formar o Biólogo como Profissional de Saúde
}

\author{
Valberto Barbosa Portoํㅗ Maria Marlene Marques Ávila²
}

\author{
${ }^{1}$ Curso de Ciências Biológicas do Centro de Ciências da Saúde da Universidade Estadual \\ do Ceará, Brasil | valberto.porto@uece.br | https://orcid.org/0000-0002-0447-9261 \\ ${ }^{2}$ Programa de Pós-graduação em Saúde Coletiva da Universidade Estadual do Ceará, \\ Brasil | marlene.avila@uece.br | https://orcid.org/0000-0002-8511-2524
}

Resumo: Introdução: A experiência de formar o biólogo como profissional de saúde, na modalidade bacharelado, já foi praticada pelo Curso de Ciências Biológicas, do Centro de Ciências da Saúde, da Universidade Estadual do Ceará, respaldada no seu projeto de criação e no seu primeiro projeto pedagógico de curso. Por outro lado, a narrativa autobiográfica experimenta avanços significativos no meio qualitativo de pesquisa científica, podendo abrir portas para a continuidade de estudos de determinadas naturezas, como sobre o biólogo como profissional de saúde, assunto recordado neste artigo. Objetivo: Relatar o modo como surgiu a experiência de um curso de biologia em formar o biólogo profissional de saúde para contribuir com a indicação de um caminho a seguir no processo de formação do biólogo licenciado, objeto de estudo da pesquisa de doutorado discutida, para este trabalho ajudar outras pessoas em atividades dessa natureza. Métodos: $O$ relato da experiência foi baseado na Metodologia Interativa, na qual usou-se da Investigação Narrativa como um dos instrumentos da triangulação, interagindo com a pesquisa documental e de campo, compondo-se neste manuscrito, de reflexão crítica, baseada em narrativa autobiográfica, para produzir os efeitos de uma generalização naturalística. Resultados: Os resultados discutidos expressam, que o curso investigado, que já praticou a formação do biólogo no campo da Saúde, não é neófito nesse empreendimento de formar biólogos como profissionais de saúde. Conclusão: A Investigação Narrativa forneceu subsídios valiosos oriundos desta prática anterior de formação, para a pesquisa de doutorado centrada na formação do biólogo licenciado neste campo de atuação, a fim de que pesquisadores possam se usufruir deste estudo para chegarem às suas próprias conclusões.

Palavras Chaves: Formação em Saúde; Ciências Biológicas; Metodologia Interativa; Pesquisa Narrativa; Autobiografia.

The Researcher and the Experience of Training the Biologist as a Health Professional

Abstract. Introduction: The experience of training the biologist as a health professional, in a bachelor's degree, has already been practiced by the Biological Sciences Course, at the Health Sciences Center, at the State University of Ceará, supported by his creation project and his first project pedagogical course. On the other hand, the autobiographical narrative experiences significant advances in the qualitative environment of scientific research, which may open doors for the continuation of studies of certain natures, such as the biologist as a health professional, a subject recalled in this article. Goal: To report how the experience of a biology course in training the professional health biologist arose to contribute with the indication of a path to follow in the process of training the licensed biologist, object of study of the doctoral research discussed, for this work to help other people in activities of this nature. Methods: The experience report was based on the Interactive Methodology, in which Narrative Investigation was used as one of the instruments of triangulation, interacting with documentary and field research, composing in this manuscript, of critical reflection, based on an autobiographical narrative, to produce the effects of a naturalistic generalization. Results: The results discussed express that the investigated course, which has already practiced the training of biologists in the field of Health, is not a neophyte in this endeavor to train biologists as health professionals. Conclusion: Narrative Research provided valuable subsidies from this previous training practice, for doctoral research focused on the training of the licensed biologist in this field, so that researchers can take advantage of this study to reach their own conclusions.

Keywords: Health Training; Biological Sciences; Interactive Methodology; Narrative Research; Autobiographical Narrative. 


\section{Introdução}

O Curso de Ciências Biológicas (CCB) do Centro de Ciências da Saúde (CCS) da Universidade Estadual do Ceará (UECE), Fortaleza, Ceará, Brasil, fundado em 14/11/1997 (UECE, 1997a) experimentou formar o bacharel em saúde pública, entre os egressos da modalidade de graduação em licenciatura plena, que optaram - amparados, inicialmente, no Projeto de Criação do Curso (UECE, 1997b) e, posteriormente, pelo Projeto Pedagógico do Curso (UECE, 2001) - pela matrícula na modalidade bacharelado, entre os anos de 1998 e 2007.

O presente estudo foi desenvolvido no intuito de ser esclarecido como se deu a inserção do CCB/CCS/UECE no campo de formação em saúde pública, com base na vivência do primeiro autor, sob o arrimo da segunda autora, sua orientadora durante o curso de doutorado, justificando textualizar-se este comunicado na primeira pessoa, por tratar-se de uma narrativa autobiográfica, sabendo-se que "a investigação narrativa tem por especificidade buscar compreender o vivido mobilizando as narrativas da experiência em primeira pessoa" (Breton, 2020, p. 13).

É por demais oportuno, não se tratando de excesso de zelo, arguir que a formação do biólogo como profissional de saúde está amparada nas normas em vigor, que, quando combinadas, Ihe conferem esta prerrogativa (Brasil, 1979; 1998; 2020).

Diante do exposto, este artigo se constitui de um recorte da Tese do primeiro autor - que procura responder ao questionamento sobre como evoluiu a proposta curricular do curso, para formar o bacharel em saúde pública? - no qual a história do pesquisador, que convergiu para a história do curso, porquanto dimensão de análise, é textualizada na forma de narrativa autobiográfica.

Por conseguinte, o objetivo deste texto é relatar sobre os aspectos da vivência do primeiro autor, conducentes ao desenvolvimento de estudos sobre a experiência de formar 0 biólogo bacharel profissional de saúde pública para contribuir com o delineamento de um caminho a seguir no processo similar de formação do biólogo licenciado, objeto de estudo que foi desenvolvido na sua pesquisa de doutorado, de modo que este trabalho possa ajudar outras pessoas a realizar atividades da mesma natureza, por meio do processo de generalização naturalística.

\section{Bases Metodológicas}

O presente relato, que é de natureza qualitativa, recorte do Estudo de Caso (Stake, 2016), desenvolvido na tese de doutorado do primeiro autor, se faz para compreender melhor o cenário deste estudo, o CCB/CCS/UECE, no tocante ao desenvolvimento da formação do biólogo como profissional de saúde, considerando-se os pressupostos filosóficos de uma pesquisa qualitativa: ontologicamente, epistemologicamente, axiologicamente e metodologicamente, usando, o pesquisador, a lógica indutiva, "a partir da estaca zero, mais do que proferida inteiramente a partir de uma teoria ou de perspectivas do investigador" (Creswell, 2014, p. 33).

Desse modo, com base na Metodologia Interativa (Oliveira, 2016), usada na tese de doutorado em recorte, a qual se ateve a viabilizar a desejada generalização assertiva (Stake, 2016), por meio de uma triangulação efetuada com os resultados colimados em pesquisa documental, investigação narrativa e pesquisa de campo, centra-se este artigo no método de investigação narrativa, por meio da técnica de narrativa autobiográfica (Galvão, 2005; 2018; Onocko Campos \& Furtado, 2008; Dutra, 2002). 
Entrementes, a técnica mobilizada na narrativa autobiográfica foi a da análise de conteúdo (Bardin, 2016), o que deu curso a um processo de reflexão crítica, organizada e diligente, para contextualizar e reconstruir, ordenadamente, a introdução da experiência inicial da formação do biólogo bacharel como profissional de saúde pública, considerando-se a trajetória do primeiro autor, convergente para a história do curso estudado, mediante um questionamento inicial, uma dimensão, categoria teórica e empíricas de análise, compondo um "quadro de coerência interna" (Costa, Neri de Souza \& Neri de Souza 2016, p. 136), buscando-se as unidades de análise (Bardin, 2016) na narrativa autobiográfica relatoriada, inerentes ao objeto de estudo da tese em recorte, para respaldar a continuidade dos estudos evidenciados nesta reflexão.

Desse modo, buscou-se demonstrar que a formação em saúde já faz parte da experiência do CCB/CCS/UECE, experiência pregressa, ora narrada, a qual está respaldada pela história do pesquisador, que vivenciou, como coordenador, o processo de criação, implantação e reconhecimento daquele, então, nascente curso (UECE, 1997c), sabendose que vivências "podem ser trazidas na lembrança do sujeito que as vivenciou" (Barbariz, 2016, p.780), resgatando o "acontecido que nos chega pela lembrança e que pode ser explicitado à medida que o sujeito expõe pela linguagem o vivenciado" (Bicudo \& Silva, 2020, p. 155), produzindo o conhecimento necessário à novas investidas investigativas.

A investigação narrativa está respaldada em Galvão (2005, p. 327; 2018, p. 2), segundo a qual "A minha vida faz-se ao contá-la e a minha memória fixa-se com a escrita; o que não ponho em palavras no papel, o tempo apaga-o. A escrita é uma longa introspecção, é uma viagem até às cavernas mais obscuras da consciência, uma lenta meditação"1 o que demonstra a importância de se textualizar uma experiência, para registrá-la como história.

O recurso à narrativa autobiográfica, que é caracterizada como um recurso metodológico em ascensão, propagado no cenário da pesquisa qualitativa, conforme se vê nos encontros científicos da temática, em nível nacional e internacional, se mostra, neste estudo, como recurso pertinente, conforme foi atestado pelo Dr. Antônio Pedro Costa, que, em e-mail ao primeiro autor, datado de 27/02/2020, assim se expressa - Na conferência que estive em Malta assisti a muitas apresentações sobre autoetnografia ... a autobiografia (narrativa na primeira pessoa) é bastante pertinente e adequada.

Desta maneira, nesta comunicação recorremos à narrativa autobiográfica, recurso atinente à investigação narrativa, que, conforme Stephens (1992) apud Galvão (2005, p. 328), engloba três componentes: "História abrange as personagens envolvidas [...] uma primeira interpretação do que é contado; Discurso forma específica como qualquer história é apresentada; Significação [...] inter-relacionamento da história e do respectivo discurso".

Ao analisar o dito por Stephens e concordando-se com Ludke (2016, p. 84), converge-se para a generalização naturalística, quando os leitores, como pesquisadores, tirarão, do lido, suas próprias conclusões:

a generalização naturalística, que vinha ao encontro do anseio legítimo de pesquisadores da educação de que os achados e as constatações de suas pesquisas possam vir a servir para a análise de problemas estudados por outros pesquisadores. De acordo com a visão de Guba (1985), corroborada por Stake (1983a, 1983b), tratando-se de situações similares, com componentes comuns, ainda que não todos, pode-se ir construindo o que consideravam uma generalização naturalística, pela análise cuidadosa das circunstâncias e também pela constância constatada de resultados semelhantes, permitindo [...] a plausibilidade da constatação. Isso poderia satisfazer a expectativa que acompanha o trabalho de todo pesquisador, sobre o reconhecimento do valor de seus achados, frutos de esforços rigorosos em torno de problemas que precisam ser resolvidos, ou pelo menos atenuados.

\footnotetext{
${ }^{1}$ Esta é uma citação de Allende (1995, apud GALVÃO, 2005, p. 327; 2018, p. 2) encontrada no seu relato autobiográfico no livro Isabel Allende - Paula. 
Entrementes, a técnica empregada parte do pressuposto de que toda história, "tem um começo, um meio e um fim" (Onocko Campos \& Furtado, 2008, p. 1092), demonstrando que 0 registro histórico se faz pela linguagem textualizada, conforme a vivência experimentada, que, "trazendo o sentimento à tona, revela também a situação, ou o contexto situacional, já que todos estão relacionados entre si” (Dutra, 2002, p. 373).

Além do até aqui exposto, para respaldar as análises necessárias, usou-se como ferramenta eletrônica o software webQDA, para armazenar no "Corpus de Dados" (Costa \& Amado, 2018, p. 32) do projeto intitulado "TESE DE DOUTORADO: CURRÍCULO DE LICENCIARURA EM CIÊNCIAS BIOLÓGICAS RELACIONADO COM A DIMENSÃO DE PROFISSIONAL DE SAÚDE INSTITUÍDA POR DISPOSITIVO LEGAL" a narrativa autobiográfica, formatada como um recordatório anamnéstico².

\section{Resultados e Discussão}

Os resultados foram obtidos com base na elaboração do quadro 1 , o qual traduziu a coerência interna desta narrativa, demonstrando o modo como foi organizada a coleta e análise dos dados, que foram discutidos, segundo o questionamento formulado, a dimensão, a categoria teórica e empíricas de análises, as observações e expectativas (Costa, Neri de Souza \& Neri de Souza 2016, p. 136), ao qual foi aplicada a técnica de análise de conteúdo (Bardin, 2016).

Quadro 1. Coerência Interna da Análise da Investigação

\begin{tabular}{|c|c|c|c|c|}
\hline $\begin{array}{l}\text { Questão de } \\
\text { Investigação }\end{array}$ & $\begin{array}{c}\text { Dimensão de } \\
\text { Análise }\end{array}$ & $\begin{array}{c}\text { Categoria Teórica } \\
\text { de Análise }\end{array}$ & $\begin{array}{c}\text { Categorias } \\
\text { Empíricas de Análise }\end{array}$ & $\begin{array}{c}\text { Observações e } \\
\text { Expectativas }\end{array}$ \\
\hline $\begin{array}{l}\text { Como surgiu a } \\
\text { proposta curricular } \\
\text { do curso, para } \\
\text { formar o bacharel } \\
\text { em saúde pública? }\end{array}$ & $\begin{array}{l}\text { Convergência } \\
\text { das Histórias: } \\
\text { Pesquisador> } \\
\text { Curso }\end{array}$ & $\begin{array}{l}\text { Antecedentes } \\
\text { Históricos }\end{array}$ & $\begin{array}{l}\text { Redimensionamento } \\
\text { da formação } \\
\text { acadêmica } \\
\text { Mestrado em Saúde } \\
\text { Pública }\end{array}$ & $\begin{array}{l}\text { Recordatório } \\
\text { Anamnéstico; } \\
\text { Narrativa } \\
\text { Autobiográfica. }\end{array}$ \\
\hline
\end{tabular}

Fonte: Adaptado e preenchido pelo primeiro autor.

A nossa expectativa era a de que a minha autobiografia, de um modo geral e sob o ponto de vista da pesquisa stricto senso, fosse necessária e suficientemente potente para nortear um novo desafio para a minha vivência de investigador, ou seja, o de delinear o caminho para formar o biólogo professor como profissional de saúde promotor da saúde na escola.

$\mathrm{Na}$ perspectiva da observação recordatória anamnéstica - de volta ao "passado [...] memória" [...] "resgatar" [...] "salvar do esquecimento" (Azevedo, 2019, p. 216 e 217) - da minha vida pregressa dedicamo-nos a textualizar os acontecimentos que não foram apagados pelo tempo e que poderiam nos ajudar a vencer o referido desafio.

De um modo particular, neste manuscrito, afunilamos o propósito norteador para a questão de investigação sobre como surgiu a proposta curricular do curso, para formar o bacharel em saúde pública [o grifo em itálico refere-se aos aspectos relacionados ao quadro 1, deste e dos parágrafos subsequentes], o que nos direcionou para a dimensão de análise Convergência das Histórias Pesquisador>Curso.

\footnotetext{
2 Técnica usada pelo autor, baseada em Azevedo (2019, p. 216 a 217) para recolher os dados, na forma de narrativa autobiográfica, sobre a dimensão de análise "O curso investigado, o CCB/CCS/UECE, atentando para a saúde como fio condutor da pesquisa".
} 
Desta dimensão de análise extraiu-se, com base na narrativa consignada na minha tese de doutorado e armazenada no corpus de dados do software webQDA, a análise do conteúdo da categoria teórica Antecedentes Históricos, subdividida - durante uma primeira leitura flutuante na pré-análise do material, complementada pela subsequente exploração do recordatório, onde se procurou alcançar o núcleo de compreensão do texto - nas categorias empíricas: Redimensionamento da formação acadêmica e Mestrado em Saúde Pública (BARDIN, 2016).

\subsection{Antecedentes Históricos}

Os Antecedentes Históricos, como categoria teórica de análise, se referem à maneira como foi criado o $\mathrm{CCB} / \mathrm{CCS} / \mathrm{UECE}$ e revelaram naquela tese, durante o recordatório anamnéstico empreendido, entre suas categorias empíricas duas, que fazem sentido serem aqui relatadas: Redimensionamento da Formação Acadêmica, documentada em UECE (1998), tendo como unidade de análise a criação do CCB/CCS/UECE e Mestrado em Saúde Pública, cuja unidade de análise é o próprio título da minha dissertação "Adequação do Curso de Ciências Biológicas da Universidade Estadual do Ceará à Formação de Biólogos com Concentração de Estudos na Área de Saúde Pública" (Porto, 2000).

\subsubsection{Redimensionamento da Formação Acadêmica}

A criação do CCB/CCS começou a fazer sentido, para mim, quando, naquela época fui nomeado pelo reitor da UECE, pela Portaria № 602/97 de 23 de abril de 1997, "para exercer as funções de Coordenador 'Pró-Tempore' do Curso de Ciências Biológicas, encarregando-se de dirigir o processo de criação, implantação e reconhecimento do referido Curso" (UECE, 1997c), haja vista o então reitor vislumbrar uma biologia forte a abrir o caminho para a criação do curso de medicina, o que se consolidou no ano de 2003.

Inicialmente, como o CCB ainda não existia, a minha tarefa de coordenador era afeita à implantação e ao reconhecimento da Habilitação Específica em Biologia do Curso de Ciências preconizada na Resolução nำ/1974 do Conselho Federal de Educação (CFE) (CFE, 1974), tipo de habilitação que deveria pertencer ao Centro de Ciência e Tecnologia (CCT) e, naqueles idos de 1997, ainda não era praticada na UECE, que já possuía as habilitações específicas de Matemática, Física e Química, implantadas pelo CCT, respectivamente, em 1978.2, 1984.2 e 1985.1, sendo posteriormente reconhecidas em 1990 (UECE, 1999).

A Habilitação Específica de Biologia ficara em modo de espera até aquele ano, de 1997, por divergências administrativas - o Curso de Ciências constituído da Licenciatura Curta e suas Habilitações Específicas pertenciam ao CCT, mas o corpo docente das disciplinas biológicas era vinculado ao CCS, desmotivando o primeiro centro de implantar um curso, cuja maioria dos professores era do segundo.

Assim, a inexistência da Habilitação Específica em Biologia inviabilizava o surgimento do $\mathrm{CCB}$, haja vista que a sua criação era parte do projeto de Redimensionamento da Formação Acadêmica, no qual se incluía a ação de Redimensionamento dos Cursos de Licenciatura em Ciências, cujo objetivo era o de "extinguir a Licenciatura Curta em Ciências [com suas habilitações plenas específicas], criando e implantando as Licenciaturas [Plenas] em Química, Física, Matemática e Biologia" (UECE, 1998, p. 13), [grifo nosso].

Como, entretanto, poder-se-ia redimensionar algo inexistente? Então, para dar curso a esta ação, o Departamento de Ensino de Graduação (DEG) da Pró Reitoria de Graduação (PROGRAD) incluiu em 1997.1, para os graduados em Licenciatura Curta do Curso de Ciências, a possibilidade de optar pela plenificação na Habilitação Específica em Biologia, ao lado das de Matemática, Física e Química, sendo que os seus primeiros alunos foram matriculados em 1997.2 (UECE, 1999). 
Consequentemente, do ato implantador do DEG/PROGRAD, houve a necessidade de designação de um coordenador, o que resultou na promulgação da já mencionada portaria, a qual me designou coordenador de curso (UECE, 1997c).

Destarte, consultando-se o Documento de Reconhecimento da Habilitação Específica em Biologia, lê-se:

A Universidade passou a oferecer a habilitação em Biologia atendendo às solicitações de muitos graduados no curso de Ciências, Licenciatura de 1응 Grau.

Com a oferta da habilitação em Biologia completava-se o quadro de opções da plenificação da Licenciatura em Ciências - Matemática, Física, Química e Biologia. Desta forma, a partir do semestre 1997.2, preencheu-se essa lacuna existente no processo de formação do professor de Ciências do $2^{\circ}$ Grau.

Assim sendo, não houve um vestibular específico para a habilitação em Biologia. Os seus alunos ingressaram na Universidade mediante concurso vestibular para o curso de Ciências, Licenciatura de $1^{\circ}$ Grau e, a partir do semestre 1997.2, passaram a optar, também, pela Habilitação Plena em Biologia, após a conclusão do curso de Licenciatura Curta (UECE, 1999, p. 3 e 4).

Ainda em 1997, antes mesmo do documento do projeto de redimensionamento ser editado, a PROGRAD realizou, no dia 05/08/1997, um encontro, do qual participei como coordenador da Habilitação Específica em Biologia, intitulado de "Redimensionamento dos Cursos de Licenciatura em Ciências" no qual, pela manhã, após a abertura, foi realizada uma mesa redonda e debates e, à tarde, foram formados Grupos de Estudos Curriculares por Assuntos, sendo traçadas as diretrizes para a criação e implantação das Licenciaturas Plenas.

Após a anunciada iniciativa da PROGRAD foi formada uma equipe de elaboração da proposta de criação do curso de Ciências Biológicas e nesse ínterim recebemos a assessoria da Coordenadoria Técnico Pedagógica (CTP) daquela pró-reitoria, a qual nos proporcionou, inclusive, consultoria externa da coordenadora do CCB da Universidade de Santa Catarina, na qual fomos incentivados a instituir um curso com as modalidades de formação de bacharelado e licenciatura, ideia que foi encampada pela equipe de elaboração da proposta de criação.

Ato contínuo, concluiu-se aquela proposta, que foi protocolada no dia 03/10/1997 (UECE, 1997b), inovando, em relação às outras licenciaturas plenas, na inclusão da modalidade bacharelado como opção de graduação, após a conclusão da licenciatura plena, na qual se incluía a profissionalização em saúde pública, compondo a unidade de análise ora relatada.

A proposta de criação foi aprovada pela Resolução № 175/97 do Conselho Universitário CONSU da UECE, em 14/11/1997 (UECE, 1997a), quando se consumou a criação do CCB/CCS/UECE, nas modalidades licenciatura e bacharelado.

\subsubsection{Mestrado em Saúde Pública}

Eu ingressei no Mestrado em Saúde Pública, da UECE, no início de 1996, apresentando o projeto de Criação de um Núcleo de Educação Ambiental naquela IES, com o intuito de formar multiplicadores educadores ambientais, haja vista a pertinência e importância do tema no campo da Biologia em interface com a Saúde. 
Todavia, como eu não estava afastado das minhas funções de professor, a minha designação, no começo de 1997, para coordenar o processo de criação, implantação e reconhecimento do Curso de Ciências Biológicas (UECE, 1997c) me fez guinar para o tema da formação do biólogo bacharel em Saúde Pública, unidade de análise desta categoria empírica, que foi algo inovador na UECE, já que as outras licenciaturas criadas - Matemática, Física, Química - não manifestaram o mesmo interesse das Ciências Biológicas nos seus processo de criação.

Assim sendo, ao mesmo tempo que participei do redimensionamento das licenciaturas, inclusa no projeto de redimensionamento da formação acadêmica (UECE, 1998, p. 13) o qual deu origem ao CCB/CCS/UECE, que se propunha a formar o licenciado, que ao se graduar poderia optar por continuar os seus estudos para obter o título de bacharel - iniciei uma pesquisa, orientado pelo Dr. Antero Coelho Neto, caracterizada pelo uso da metodologia "da Pesquisa Social [de Gil, (1991)] [...] associada ao Planejamento Estratégico para a Melhoria da Qualidade [de Coelho Neto (1996)] (apud, Porto, 2000, p. 19).

O intuito era o de saber se era adequado este caminho de formar o biólogo bacharel, concentrando os seus estudos na área da Saúde Pública, portanto, claramente, um profissional de saúde, chegando-se à conclusão que sim, oportunizando analisar este processo de formação para usar os dados coletados, que foram explicitados em nova categoria teórica de análise, Formação do Biólogo Bacharel Profissional de Saúde, incluída na minha tese de doutorado, a ser tematizada em outro artigo, como uma experiência exitosa.

\section{Considerações Finais}

O primeiro autor e sua orientadora segunda autora deste artigo, consideram pertinente a apresentação deste manuscrito neste 10 Congresso Ibero-Americano de Investigação Qualitativa (CIAIQ, 2021), por ser um recorte de uma pesquisa qualitativa de Estudo de Caso - que teve como objeto de estudo o processo de formação do biólogo professor como profissional de saúde, levada a efeito no Programa de Pós Graduação em Saúde Coletiva da UECE - inteiramente compatível com o objetivo do congresso, o qual incentiva a "submissão de artigos científicos que foquem diversos campos de aplicação na Investigação Qualitativa, desde a Educação à Saúde...", conforme estampado na página de apresentação do congresso, cuja url é https://ciaiq.ludomedia.org.

A metodologia qualitativa é o princípio norteador deste artigo, que versa sobre a investigação narrativa como método de análise, que foi realizada com base na técnica da narrativa autobiográfica do primeiro autor, para compor o procedimento metodológico mais amplo, o da Metodologia Interativa, levada a efeito na sua pesquisa de doutorado, para a triangulação dos achados compartilhados com uma pesquisa documental sobre as normas e currículos praticados nos cursos de licenciatura em Ciências Biológicas e com a pesquisa de campo, usando a técnica da Roda de Conversa, tendo como participantes os formadores do CCB/CCS/UECE, tudo estando carregado no corpus de dados de um projeto, constante do software webQDA, usado como suporte às análises e interpretações requeridas.

No artigo, ao se questionar como surgiu a proposta curricular do curso para formar o bacharel em saúde pública, teve-se o objetivo de relatar sobre a experiência pregressa do CCB/CCS/UECE de formar o biólogo bacharel profissional de saúde pública, para contribuir com a indicação de um caminho a seguir no processo similar de formação do biólogo licenciado, assunto referenciado internacionalmente: a exemplo cita-se na Universidade do Minho, Braga, Portugal e na Universidade de Valencia, Valencia, Espanha, discussão sobre a promoção da saúde na escola, em artigos de José Precioso, Marta Talavera e Valentin Gavídia. 
Em continuação aos estudos de mestrado sobre o biólogo bacharel profissional de saúde, experiência de formação comunicada neste artigo, o doutorado foi um novo desafio, no qual estudou-se a perspectiva de formação do licenciado, também para atuar como profissional de saúde.

O legado daquela trajetória inicial de formação do biólogo bacharel teve a potência de proporcionar subsídios valiosos na elaboração da pesquisa de doutorado, bem como poderá ajudar outras pessoas a realizar atividades similares pela via da enfatizada generalização naturalística, o que julgamos merecer a sua divulgação, internacionalmente, no seio dos pesquisadores qualitativos.

\section{Referências}

Azevedo, F. F. S. (2019). Dicionário Analógico da Língua Portuguesa, ideias afins/thesaurus. 3. ed. Rio de Janeiro: Lexiton Editora Digital Ltda.

Barbariz, T. A. M. (2016). Descrição, análise e interpretação de vivências em pesquisa qualitativa fenomenológica. Porto: Atas do CIAIQ, 779-784.

Bardin, L. (2016). Análise de Conteúdo. São Paulo: Edições 70.

Bicudo, M. A. V., \& Silva, A. A. da. (2017). Análise de Descrições de Vivências em Situações de Constituição de Conhecimento. In Costa, A. P., Sánchez-Gómez, M. C. Cilleros, M. V., A prática da Investigação Qualitativa. Aveiro: Ludomedia, 153-178.

Brasil., Senado Federal. (1979). Lei oㅡ 6.684 de 03/09/1979. Brasília: Congresso Nacional.

Brasil., Conselho Nacional de Saúde. (1998). Resolução n ${ }^{0}$ 287/08/1998. Relaciona as 14 (quatorze) categorias profissionais de saúde de nível superior para fins de atuação no Conselho Nacional de Saúde. Brasília: Diário Oficial da União, Conselho Nacional de Saúde.

Brasil., Ministério da Saúde. (2020). Portaria № 639, de 31/03/2020: Ação Estratégica "O Brasil Conta Comigo - Profissionais da Saúde". Brasília: Diário Oficial da União, Ministério da Saúde.

Breton, H. (2020). Investigação narrativa: entre detalhes e duração. Boa Vista: Revista Educação, Pesquisa e Inclusão, 1(1), 12-22.

Conselho Federal de Educação (CFE). (1974). Resolução oㅡ 30 de 11/07/1974. Fixa os mínimos de conteúdo e duração na organização do curso de licenciatura em Ciências. Brasília: CFE.

Costa, A. P., Neri de Souza, F., \& Neri de Souza, D. (2016). Investigação Qualitativa Inovação, Dilemas e Desafios. Volume 1. Aveiro: Ludomedia.

Costa, A. P. Amado, J (2018). Análise de Conteúdo Suportada por Software. Aveiro: Ludomedia.

Creswell, J. W. (2014). Investigação qualitativa e projeto de pesquisa: escolhendo entre cinco abordagens. $3^{\mathrm{a}}$ ed. Porto Alegre: Penso.

Dutra, E. (2002). A narrativa como uma técnica de pesquisa fenomenológica. Natal: Revista Estudos de Psicologia. 7(2), 371-378.

Freitas, D. de; \& Galvão, C. (2007). O uso de narrativas autobiográficas no desenvolvimento profissional de professores. Rio de Janeiro: Ciências \& Cognição, Ano 04, Vol 12.

Galvão, C. (2005). Narrativas em Educação. Bauru: Revista Ciência \& Educação, 11(2), 327-345.

Galvão, C. (2018). Narrativas na Educação. Apresentação em PDF. Lisboa: Instituto de Educação/Universidade de Lisboa.

Lüdke, M. (2016). Educação e pesquisa qualitativa no Brasil. In Neri de Souza, D., Costa, A. P., \& Neri de Souza, F. (Organizadores). Investigação Qualitativa: Inovação, Dilemas e Desafios. Oliveira dos Azimeis: Ludomedia, 77-104.

Oliveira, M. M. (2016). Como fazer pesquisa qualitativa. 7. ed. Petrópolis: Vozes.

Onocko Campos, R. N. \& Furtado, J. P. (2008). Narrativas: utilização na pesquisa qualitativa em saúde. São Paulo: Revista de Saúde Pública, 42(6), 1090-1096. 
Vol. 8 | Investigação Qualitativa em Saúde: Avanços e Desafios

Porto, V. B. (2000). Adequação do Curso de Ciências Biológicas da Universidade Estadual do Ceará à Formação de Biólogos com Concentração de Estudos na Área de Saúde Pública. Dissertação de Mestrado. Fortaleza: Universidade Estadual do Ceará.

Stake, R. E. (2016). A Arte de Investigação com Estudos de Caso. Lisboa: Fundação Calouste Gulbenkian.

Universidade Estadual do Ceará. (1997a). Resolução № 175/97 - CONSU. Aprova a criação dos cursos que indica. Fortaleza: Reitoria da UECE.

Universidade Estadual do Ceará. (1997b). Proposta de Criação do Curso de Ciências Biológicas Modalidades: Licenciatura Plena e Bacharelado. Fortaleza: Coordenação do Curso de Ciências Biológicas.

Universidade Estadual do Ceará. (1997c). Portaria № 602/97/97 - FUNECE. Designa o Coordenador Pró-Tempore do Curso de Ciências Biológica. Fortaleza: FUNECE.

Universidade Estadual do Ceará. (1998). Projeto de Redimensionamento da Formação Acadêmica na UECE. Fortaleza: UECE/Pró-Reitoria de Graduação.

Universidade Estadual do Ceará. (1999). Reconhecimento do Curso de Ciências Licenciatura Plena com Habilitação em Biologia. Fortaleza: Curso de Ciências Biológicas.

Universidade Estadual do Ceará. (2001). Projeto Pedagógico Curso de Ciências Biológicas. Fortaleza: Curso de Ciências Biológicas. 